\title{
Simulating the Electron Transmission with Different Defective Carbon Nanotubes
}

\author{
R. NIZAM ${ }^{a, *}$, S.M.A. RIZvi ${ }^{b}$ AND A. AzAM ${ }^{c}$ \\ ${ }^{a}$ Centre of Excellence in Material Science, Applied Physics Aligarh Muslim University, Aligarh, India \\ ${ }^{b}$ Department of Mechanical Engineering, Aligarh Muslim University, Aligarh, India \\ ${ }^{c}$ Centre of Nanotechnology, King Abdulaziz, Kingdom of Saudi Arabia
}

(Received September 5, 2011; revised version February 29, 2012; in final form March 29, 2012)

\begin{abstract}
2. The transmissions of forty-eight carbon nanotube geometries to form twenty-four intramolecular junctions between every two carbon nanotubes and two simple carbon nanotubes are also taken for investigating numerically. These forty-eight carbon nanotubes form three different kinds of intramolecular junctions, which mean that each sixteen carbon nanotubes are identical to either sixteen carbon nanotubes in all respect, except three different intramolecular junctions in between them. The three intramolecular junctions, named as circumferential defective carbon nanotubes, grouped defective carbon nanotubes and distributed defective carbon nanotubes. The nature of electronic states at the interfaces and in the semiconductor section is analyzed with the help of tight-binding method. These quantum transmissions have been compared among the different defective carbon nanotubes and have been correlated with the pentagon and heptagon that formed in the intramolecular junction. It has been simulated that each pair of semiconductor-semiconductor carbon nanotubes, semiconductor-metallic carbon nanotubes and metallic-metallic carbon nanotubes transmission affect on the presence of junctions pentagonsheptagons. The least deviations transmission is observed in the circumferential defective carbon nanotubes rather than in other two and the maximum deviations are seen in the grouped defective carbon nanotubes irrespective of the joining of the carbon nanotube interface.
\end{abstract}

PACS: $73.22 .-\mathrm{f}$

\section{Introduction}

Single-walled carbon nanotubes (SWNTs) are made of graphite hexagonal carbon-atom lattice shaped into a cylinder. It has drawn a great deal of interests because of their fundamental research importance with tremendous potential technical applications [1]. They may be possibly future molecular electronic devices such as room temperature single electron and field-effect transistors and rectifiers $[2-6]$. Depending on its helicity and diameter, an SWNT can be either a semiconductor or a metal [7-10]. Thus two segments of SWNTs with different diameters and helicities can be connected by introducing the pentagon-heptagon defects into the perfect hexagonal network. It will create the metal-semiconductor (M-S), semiconductor-semiconductor (S-S), or metalmetal (M-M) intermolecular junctions (IMJs) which may be possibly the building blocks for much smaller electronic devices than those with conventional semiconductor technology.

In metallic perfect carbon nanotube, the total transmission of particular armchair carbon nanotube $(5,5)$ is taken whose the Fermi energy $(E=0)$ is equal to two. There are only two sub-bands per spin at the Fermi energy, independent of carbon nanotube diameter and chirality. At about $1.75 \mathrm{eV}$, the transmission jumps from two to six units because the sub-bands have a degeneracy of two as shown with green colour line in Fig. 1. The total number of sub-bands increases with increasing

* corresponding author; e-mail: rashid.nizam@gmail.com

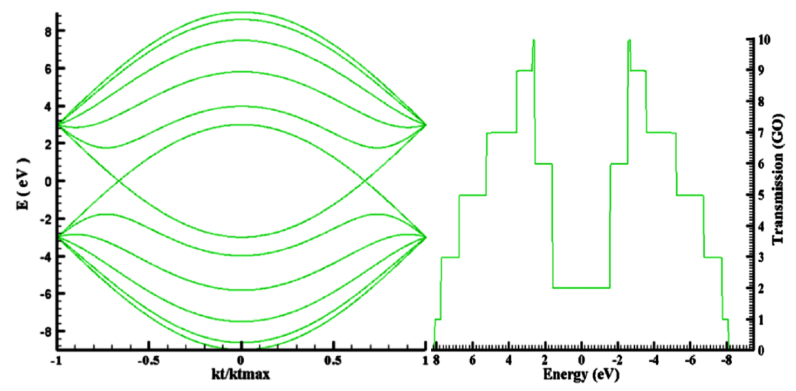

Fig. 1. The sub-bands of carbon nanotube $(5,5)$ and its transmission function.

nanotube diameter as the number of quantum numbers arising from quantization of the electron wave function around the nanotube circumference also becomes larger.

It is important to note that in case of metallic carbon nanotube, the conductance and valence bands have mirror symmetry at the Fermi energy level where the sub-bands are denoted by the lines cross (which occurs at the zero of energy in Fig. 1), and it is independent of nanotube diameter. These sub-bands are called crossing sub-bands. In case of semiconducting zigzag carbon nanotube, similar transmissions are shown as represented in Fig. 2 which are also in steps but they become zero at the Fermi energy level.

From 1995, the electronic properties of the SWNT IMJs have been growing number of experimental along with theoretical studies $[11,12]$. It is pointed out that a pentagon-heptagon pair with a symmetry axis nonparal- 


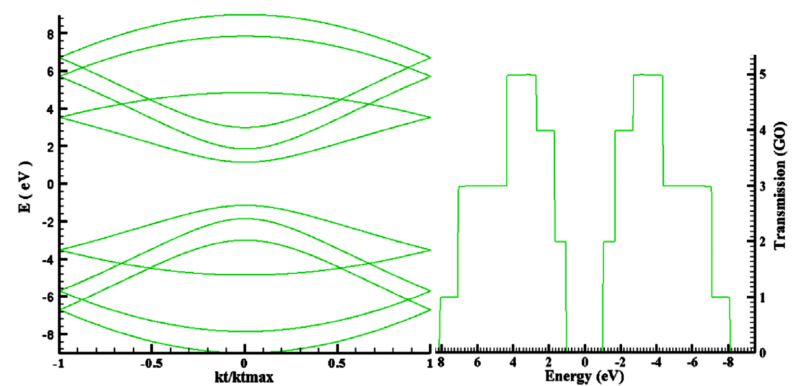

Fig. 2. The sub-bands of carbon nanotube $(5,0)$ and its transmission function.

lel to the tube axis could modify the nanotube helicity by one unit from $(n, m)$ to $(n+1, m-1)$ by Chico et al. $[13,14]$. Some researchers gave general features of the IMJs in the zigzag configuration [15]. Such kinds of studies recommended that these arrangements could function as nanoscale electronic devices prepared entirely of carbon atoms. Even though great progress in the IMJ research has been made, yet there are lots of experimental with theoretical uncertainties about the close relationships between their physical properties and the corresponding geometrical structures.

In recent times, Ouyang et al. calculated the $(21,-2)$ / $(22,-5)$ and $(11,8) /(9,6)$ IMJs by using scanning tunneling microscopy (STM), which gave clear experimental confirmation of the SWNT IMJs [16]. He explained that the M-S IMJ has an electronically sharp interface without the localized junction states while a more extended interface in addition to low-energy localized states are found in the M-M IMJ [16]. They proposed several atomic models to simulate their observed experimental data results.

\section{Model}

To build models of the IMJs, the defected carbon nanotube DCNT $(n, m)$ indices that correspond to a specific combination of $d$ and $\theta$ [17] were determined by using an iterative projection matching method [18]. IMJs can be understood by interposing one or multipose topogical junctions (IMJs) formed by interposing one or multiple topologic pentagon-heptagon (5-7) defects in the normal hexagonal structure of two carbon nanotubes segments with different helicity. Some of the theoretical studies of the electronic properties of model DCNT propose that these structures could function as molecular-size $\mathrm{M}-\mathrm{S}$, $\mathrm{M}-\mathrm{M}$, or S-S building blocks with healthy solid-state behavior.

Taking the same idea here, we considered forty-eight carbon nanotube geometries to form twenty-four intramolecular junctions between every two carbon nanotubes. These carbon nanotubes forming three different kinds of the Stone-Wale defect form intramolecular junctions, which mean each sixteen carbon nanotubes, are identical to either sixteen carbon nanotubes in all re- spect, except three different intramolecular junctions in between them.

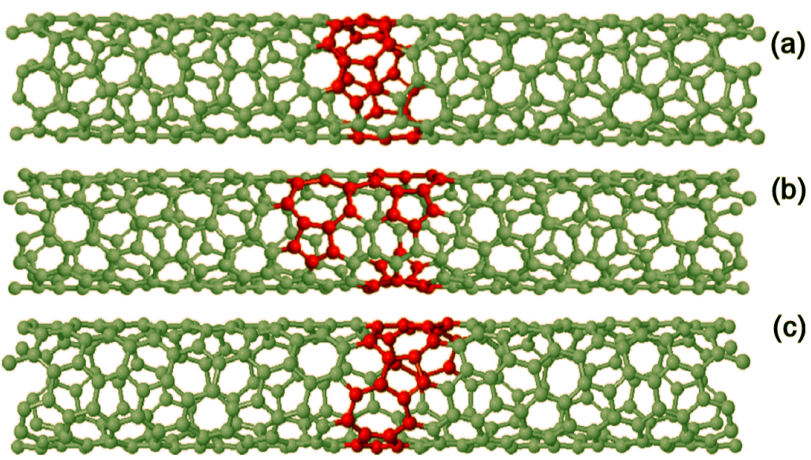

Fig. 3. Intramolecular junction of circumferential (a), distributed (b), and grouped (c) DCNT between $6 \times 3$ and $6 \times 3$, respectively.

The three intramolecular junctions named as circumferential defective carbon nanotubes, grouped defective carbon nanotubes and distributed defective carbon nanotubes as shown in Fig. 3 with the pair of indices $(n, m)$. In the circumferential defective carbon nanotubes the pentagon and heptagon is formed more in the circumference of the joining of the two carbon nanotubes as shown in Fig. 3a while in the distributed defective carbon nanotubes these pentagon and heptagon are arranged in the different site on the carbon nanotube as shown in Fig. 3b. In the grouped defective carbon nanotubes the pentagon and heptagon grouping is main formed in one side of middle of junction of carbon nanotube as shown in Fig. 3c. Defective carbon nanotubes, the pentagon and heptagon, is formed at different sites of the carbon nanotubes.

The configuration of different carbon nanotubes are used in the paper with three different intramolecular junctions as follows but only pictures of each circumferential DCNT are shown because of large number of DCNTs.

1. Semiconductor $(5,3)$ - semiconductor $(5,3)$ radii, $2.74 \AA$ and 266 number of atoms respectively. As shown in Fig. 4a.

2. Semiconductor $(5,4)$ - semiconductor $(5,4)$ radii, $3.06 \AA$ and 294 number of atoms respectively. As shown in Fig. $4 \mathrm{~b}$.

3. Metallic $(6,3)$ - metallic $(6,3)$ radii, $3.11 \AA$, $3.11 \AA$, respectively and 304 number of atoms. As shown in Fig. 3a and Fig. 4c.

4. Semiconductor $(6,4)$ - semiconductor $(6,4)$ radii, $3.11 \AA$ and 332 number of atoms respectively. As shown in Fig. 4 d.

5. Semiconductor $(6,4)$ - metallic $(6,3)$ radii, $3.11 \AA, 3.41 \AA$, respectively and 314 number of atoms. As shown in Fig. 4e. 

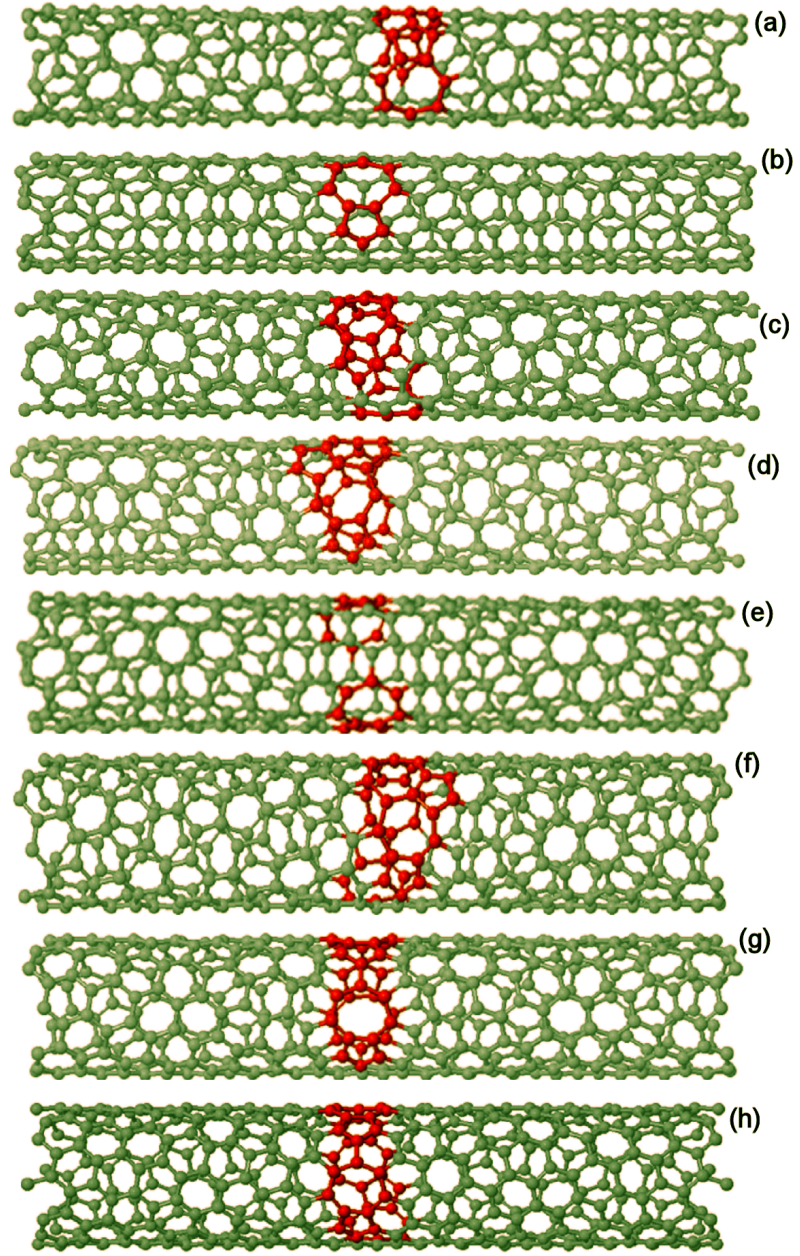

Fig. 4. Intramolecular junction of circumferential DCNT between $(\mathrm{a})-(5,3)$ and $(5,3),(\mathrm{b})-(5,4)$ and $(5,4),(\mathrm{c})-(6,3)$ and $(6,3),(\mathrm{d})-(6,4)$ and $(6,3),(\mathrm{e})-(6,4)$ and $(6,4),(\mathrm{f})-(7,3)$ and $(7,4)$, (g) $-(7,4)$ and $(7,4)$, and $(\mathrm{h})-(8,4)$ and $(8,4)$, respectively.

6. Semiconductor $(7,3)$ - metallic $(7,4)$ radii, $3.48 \AA, 3.77 \AA$, respectively and 353 number of atoms. As shown in Fig. 4f.

7. Metallic $(7,4)-$ metallic $(7,4)$ radii, $3.77 \AA$, $3.77 \AA$, respectively and 364 number of atoms. As shown in Fig. 4g.

8. Semiconductor $(8,4)$ - semiconductor $(8,4)$ radii, $4.14 \AA$ and 406 number of atoms respectively. As shown in Fig. 4h.

In some given pairs of indices, all the three defective carbon nanotubes form the same defective structure that makes the transmission through each defective carbon nanotube exactly the same. It is because the defective carbon nanotubes have the same quantum states for fixed indices which match the experimental data. For the semiconducting and metallic portions, various indices are used such as $(5,5)$ and $(7,4)$, respectively. Here each nanotube $20 \AA$, almost the same radii, is used with total length $40 \AA$. The energy grid of different nanotube is used $12 \mathrm{eV}$ and $\pm 2 \mathrm{eV}$ at the lower and upper bound of nanotube respectively. SWNT segments with these indices can be joined faultlessly along a common axis using different configurations of 5-7 defects.

Two perfect carbon nanotubes are taken according to Rashid et al. (armchair and zigzag carbon nanotubes) to explain the transmission through them so that simulation of DCNT can be understood easily [22].

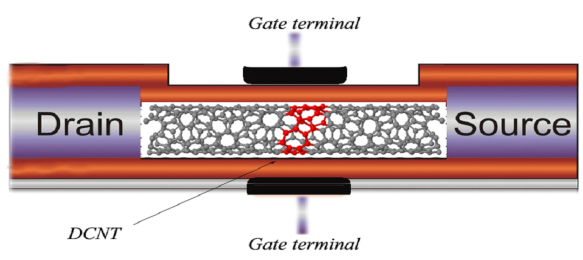

Fig. 5. MOSFET structure involves a channel made of DCNT structure.

The simulation of carbon nanotubes transmission consisting of MOSFET like structure involves a channel made of CNT structure as shown in Fig. 5. It is connected with two electrodes: a source and a drain. An insulating thin film, usually silicon dioxide, separates the channel, source, and drain from a third electrode called the gate $(\mathrm{G})$, as pictured schematically. By applying a voltage $V_{\mathrm{gs}}$ between the gate and source, the conductance of the semiconducting channel can be modulated. Charge carriers (electrons or holes) traveling between source and drain encounter a material- and structure-dependent energy barrier in the bulk of the semiconductor. Each time different DCNT is used in the form of channel in the MOSFET structure.

\section{Simulation details}

The electronic structure of a CNT can be acquired from that of graphene. The wave vector related with the chiral vector $C_{\mathrm{h}}$, in the circumferential direction gets quantized. On the other wave-vector associated, the direction of the translation vector $T$ along the CNT axis remains continuous for an infinite carbon nanotube. These are the boundary conditions of the carbon nanotube.

Various low-energy structural models are shown (Figs. 3 and 4), which have been optimized using molecular mechanics energy minimization. It is possible to evaluate the possibility of these atomic models by calculating the local electronic density of states (DOS) and comparing these with experiment [16]. But very few experiments have been done to join the different various carbon nanotubes. The simulation of each intramolecular junction is done, that contain 1000 energy points included on the both grids. The system is described by a simple tight-binding Hamiltonian with constant nearest neighbour. 
In this study, the same parameter of hexagon of carbon nanotube is used for the bonds of the pentagon-heptagon pairs of defective carbon nanotube. For a more accurate model, parameters fitted from tight-binding calculations should be used. The Hamiltonian is divided into two blocks corresponding to two semiconductors nanotube or semiconductor-metallic nanotubes or two metallic nanotubes as shown in different figures. The DOSs is computed using the standard recursion technique [20]. The transmission function can be expressed in terms of the Green functions of the carbon nanotubes and coupling of the carbon nanotubes to the contact electrodes [16-18]:

$$
T=\operatorname{Tr}\left[\Gamma_{1} G^{\mathrm{r}} \Gamma_{2} G^{\mathrm{a}}\right],
$$

where $G^{\mathrm{r}, \mathrm{a}}$ are the retarded and advanced Green functions of the carbon nanotube and $\Gamma_{1,2}$ are functions that describe the coupling of the carbon nanotube to the electrodes. Firstly we calculate the Green function of the carbon nanotube of the whole system

$$
\epsilon-H=I \text {, }
$$

where $\epsilon=E+\mathrm{i} \eta$ with arbitrary small and $I$ is the identity matrix. Here the Hamiltonian of the system is expressed in a discrete real space matrix representation that consists of the carbon nanotube and the semi-infinite electrodes. The above Green functions are divided into sub-matrices that correspond to the individual subsystems,

$$
\begin{aligned}
& \left(\begin{array}{ccc}
G_{\mathrm{L}} & G_{\mathrm{LC}} & G_{\mathrm{LCR}} \\
G_{\mathrm{CL}} & G_{\mathrm{C}} & G_{\mathrm{CR}} \\
G_{\mathrm{LRC}} & G_{\mathrm{RC}} & G_{\mathrm{R}}
\end{array}\right) \\
& =\left(\begin{array}{ccc}
\epsilon-H_{\mathrm{L}} & h_{\mathrm{LC}} & 0 \\
h_{\mathrm{LC}}^{\dagger} & \epsilon-H_{\mathrm{C}} & h_{\mathrm{CR}} \\
0 & h_{\mathrm{CR}}^{\dagger} & \epsilon-H_{\mathrm{R}}
\end{array}\right) .
\end{aligned}
$$

Here the matrix $\epsilon-H_{\mathrm{C}}$ represents the finite isolated carbon nanotube, $\epsilon-H_{\mathrm{L}, \mathrm{R}}$ represents the coupling matrices that will be nonzero only for adjacent points in the carbon nanotube and the electrodes, respectively. Then $G_{\mathrm{C}}$ can be easily calculated [18]:

$$
G^{\mathrm{r}, \mathrm{a}}(E)=\left[\epsilon-H_{s}-\Sigma_{1}^{\mathrm{r}, \mathrm{a}}(E)-\Sigma_{2}^{\mathrm{r}, \mathrm{a}}(E)\right]^{-1},
$$

where $\Sigma_{1}^{\mathrm{r}, \mathrm{a}}=h_{\mathrm{LC}}^{\dagger} g_{\mathrm{L}} h_{\mathrm{LC}}$ and $\Sigma_{2}^{\mathrm{r}, \mathrm{a}}=h_{\mathrm{CR}}^{\dagger} g_{\mathrm{R}} h_{\mathrm{CR}}^{\dagger}$ defined as the self-energy terms due to the semi-infinite electrode and $g_{\{\mathrm{L}, \mathrm{R}\}}=\left(\epsilon-H_{\{\mathrm{L}, \mathrm{R}\}}\right)^{-1}$ are electrodes' Green's functions. The self-energy terms can be considered as effective Hamiltonians that arise from the coupling of the carbon nanotube with the electrodes. By knowing the Green functions, the coupling functions $\Gamma_{1,2}$ can be easily obtained as [18]:

$$
\Gamma_{1,2}(E)=\mathrm{i}\left[\Sigma_{1,2}^{\mathrm{r}}(E)-\Sigma_{1,2}^{\mathrm{a}}(E)\right]
$$

where $\Sigma_{1,2}^{\mathrm{a}}(E)$ and $\Sigma_{1,2}^{\mathrm{r}}(E)$ are the advanced Hermitian self-energy and the retarded Hermitian self-energy which is the conjugate of the advanced Hermitian self-energy [19-21].

\section{Results and discussion}

The distinctive band structure of metallic carbon nanotubes is partially responsible for their excellent current carrying capacity that makes different from the electronic band structure of conventional metals. The transmission versus energy is an important feature of the electronic band structure of metallic carbon nanotubes that are necessary to understand their current-voltage characteristics. The numbers of carbon atoms chosen are taken large enough to avoid the effects of the dangling $\pi$ bonds at the edges of the junctions. We performed a tight binding (TB) calculation at nearly each intramolecular junction of defective carbon nanotubes.

The transmission versus energy through different intramolecular junction carbon nanotubes are shown in Figs. $6-13$. The band structure shows various sub-bands that arise from quantization of the wave vector around the circumference of the carbon nanotube irrespective of nature of carbon nanotube structure. The total transmission at a given electron energy is equal to the electron transmission probability times the number of channels but in case for perfect carbon nanotube the transmission without scattering (i.e. transmission probability equal to 1) is simply equal to the number of channels. So the total transmission shows different curves when a sub-band opens or closes. The magnitude of the change in transmission at these curves represents the sub-band degeneracy of the band structure. For example, the different colour lines are not degenerate sub-bands as shown in the figure and each contributes one channel for transmission in case of defective carbon nanotube.

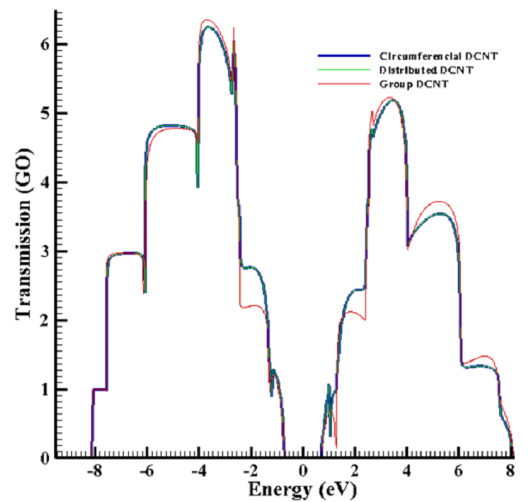

Fig. 6. Transmission through the intramolecular junctions DCNT between $(5,3)$ and $(5,3)$.

For example, the different colour lines and the dashed lines are not degenerate sub-bands as shown in the figures and each contributes one channel for transmission in simple carbon nanotube and defective carbon nanotube, respectively.

For simpler understanding the effect of the pentagonheptagon on transmission in the defective carbon nanotube only the local defective part transmission are taken. So the transmission is simulated at the junction of carbon nanotubes (DCNTs) where the pentagon-heptagons are found in the defective carbon nanotube configuration.

Some more important feature that come into light about the defective carbon nanotube interfering of the 


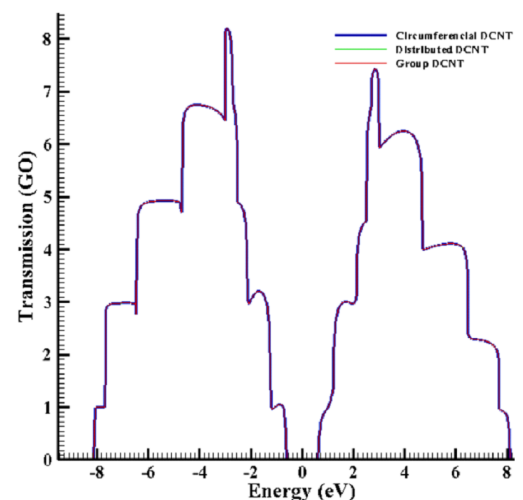

Fig. 7. Transmission through the intramolecular junctions DCNT between $(5,4)$ and $(5,4)$.

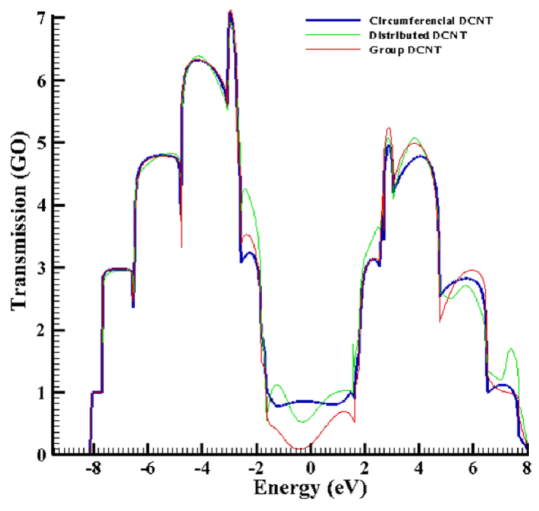

Fig. 8. Transmission through the intramolecular junctions DCNT between $(6,3)$ and $(6,3)$.

junction by these simulations as follows:

1) The position of the localized states above and below the Fermi energy level may be controlled with the distribution of the defect pairs (pentagons-heptagons) and the hexagons around the defects in the defected carbon nanotube.

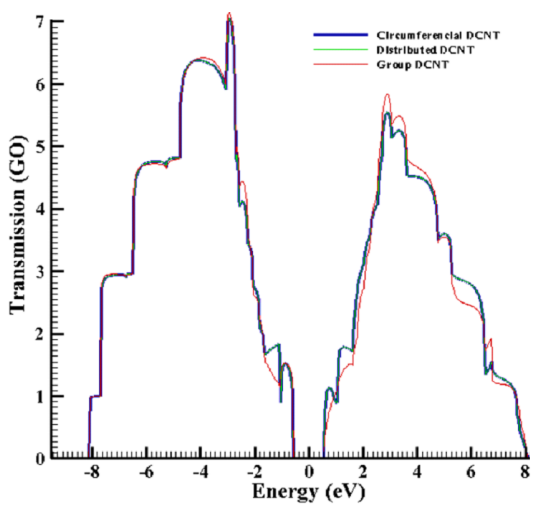

Fig. 9. Transmission through the intramolecular junctions DCNT between $(6,4)$ and $(6,3)$.

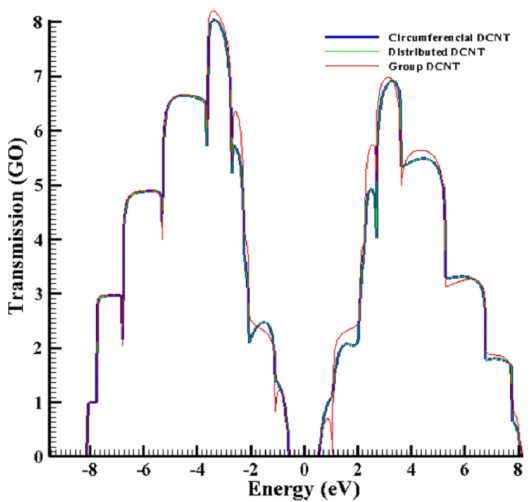

Fig. 10. Transmission through the intramolecular junctions DCNT between $(6,4)$ and $(6,4)$.

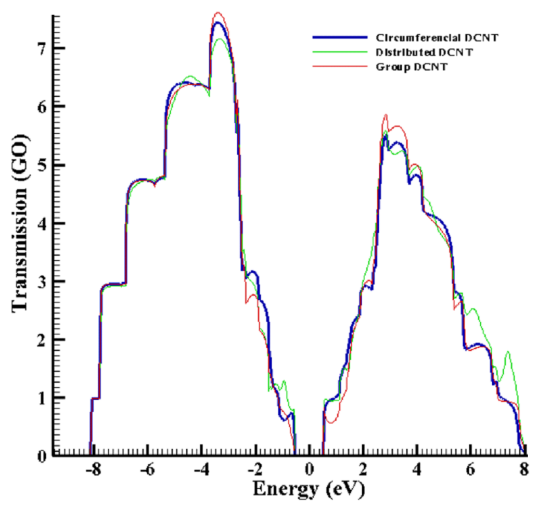

Fig. 11. Transmission through the intramolecular junctions DCNT between $(7,3)$ and $(7,4)$.

2) The more hexagons around the defects, the less curved are obtained in the transmission and become stepwise transmission.

3) Due to the complex correlation involving in the defect structures and the electronic properties, other factors such as the bond lengths of topological defects may be

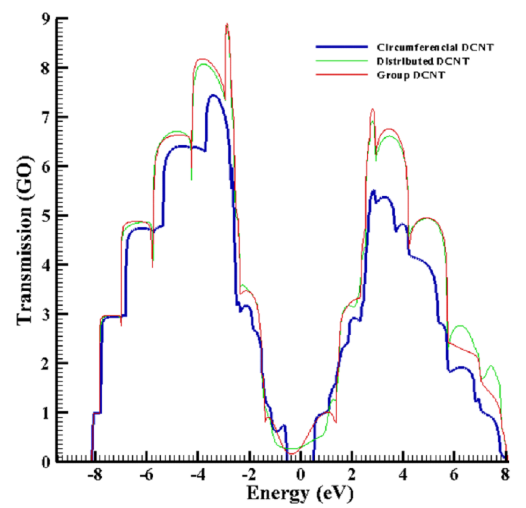

Fig. 12. Transmission through the intramolecular junctions DCNT between $(7,4)$ and $(7,4)$. 


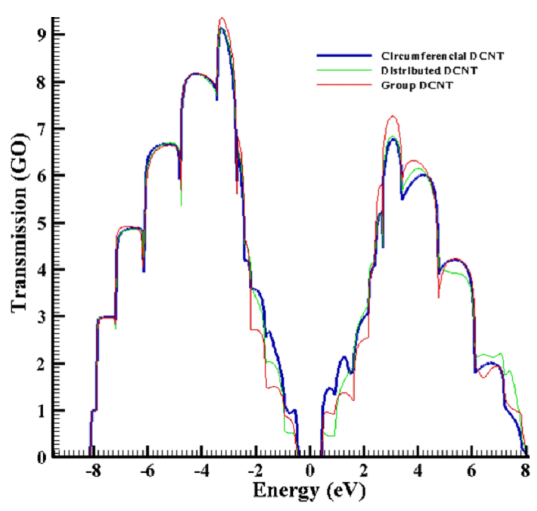

Fig. 13. Transmission through the intramolecular junctions DCNT between $(8,4)$ and $(8,4)$.

also taken into account.

4) Each pair of semiconductor-semiconductor carbon nanotubes, semiconductor-metallic carbon nanotubes and metallic-metallic carbon nanotubes transmission affect on the presence of pentagons-heptagons. It is observed that there are less fluctuations in the circumferential defective carbon nanotubes than in other two, among all types of defective carbon nanotubes.

5) The transmission coefficient of conduction band always simulated less than the transmission coefficient of valence band in each intramolecular junction irrespective of the joining of carbon nanotubes.

6) The maximum fluctuations in transmission are seen in the grouped defective carbon nanotubes and the least are obtained circumferential defective carbon nanotubes irrespective of the joining of the carbon nanotube interface.

7) Some joining of the carbon nanotubes have the same quantum configuration structure irrespective of the defect following the three degeneracy states and thus obtain the same transmission for the all the defective carbon nanotubes as in Fig. 7. On the other hand, in some case one of degeneracy states gets separated out from the other degeneracy states as shown in Figs. 9 and 10, thus there follow two different transmissions for a given pair of defective carbon nanotubes indices. One transmission obtained from one state is different for other, the same two given degeneracy states of defective carbon nanotube degeneracy states.

8) It is interesting to note that when two chiral metallic nanotubes are joined by different defects, the transmission is reduced in each defective carbon nanotube and even in the circumferential defective carbon nanotube case, it comes to zero as shown in Figs. 6, 7, 9-11, and 13, respectively. This is because the pentagon-heptagons are more effective for this particular quantum configuration structure. While in other case all defective carbon nanotubes affect on the transmission, but circumferential defective carbon nanotubes affect least and the grouped defective carbon nanotubes are the most of the type quantum configuration structure.
The total number of sub-bands increases with increasing nanotube diameter, as the number of quantum numbers arising from quantization of the electron wave function around the nanotube circumference also becomes larger. The occupied valence states of the IMJ seem to be mainly affected by the presence of the shared bond between pentagon-heptagons in each defective carbon nanotube. In each case of defective carbon nanotube, the greater is the number of pentagon-heptagons found in the defective carbon nanotube configuration, the greater variation is observed in occupied valences states of irrespective defective carbon nanotube. However, the number of the defective states below the Fermi level does not equal the number of the shared bonds between the pentagon-heptagons.

It is observed that the conductance and valence bands have different shapes transmission across the Fermi energy level where the sub-bands are denoted by the lines cross and become energy zero in each chiral semiconductor carbon nanotube with small diameter of carbon nanotube. These sub-bands are called crossing sub-bands. The different shapes transmission across the Fermi energy level lie because defective carbon nanotube have bond between pentagon-heptagons at the Fermi level and carbon nanotubes are extremely sensitive toward the bonding between the carbon atoms.

The location of the wave vector $k$ of defective carbon nanotube changes with carbon nanotube chirality where the crossing occurs. There are only two sub-bands per spin at the Fermi energy, independent of carbon nanotube diameter and chirality. It should be noted that the chiral semiconducting sub-bands are also referred to as non-crossing sub-bands as well as crossing sub-bands depending on the indices $(n, m)$. For a given indices $(n, m)$ carbon nanotube, $n-m$ is exactly divisible by 3 , then the carbon nanotube is metallic where carbon nanotubes with residuals 1 and 2 of the division $n-m$ by 3 are semiconducting.

For a $(n, m)$ carbon nanotube, $n-m$ is exactly divisible by 3 , then the carbon nanotube is metallic where carbon nanotubes with residuals 1 and 2 of the division $n-m$ by 3 are semiconducting. It is observed that the transmission of defective carbon nanotubes remains constant at the Fermi level for given pair of indices in each chiral carbon nanotube. Every chiral semiconductor carbon nanotube transmission become zero at the Fermi energy level in the transmission-energy graph irrespective on the number of carbon nanotube defects while the transmission of chiral metallic carbon nanotubes may get zero depending on the number of pentagons-heptagons in the defective carbon nanotube. The greater number of pentagons-heptagons in the defective carbon nanotube leads to the transmission zero and affects the conductivity of the carbon nanotube and vice versa.

\section{Conclusion}

It is observed that the carbon nanotube is very sensitive towards the atomic structure of their atoms in 
the carbon nanotubes, and position of the localized states above and below the Fermi energy level may be controlled with the distribution of the defect pairs (pentagons-heptagons) and the hexagons around the defects in the defected carbon nanotube. As the configuration of carbon nanotubes contain more hexagons around the defects, the less curved ones are obtained in the transmission and become stepwise transmission. The configuration of defective carbon nanotubes involve the complex correlation, so the bond lengths of topological defects are taken into account. Besides each pair of semiconductor-semiconductor carbon nanotubes, semiconductor-metallic carbon nanotubes and metallicmetallic carbon nanotubes transmission affect on the presence of pentagons-heptagons. The transmission coefficient of conduction band always simulated less than the transmission coefficient of valence band in each intramolecular junction irrespective of the joining of carbon nanotube. The maximum variations in transmission are seen in the grouped defective carbon nanotubes and the least is obtained circumferential defective carbon nanotubes irrespective of the joining of the carbon nanotube interface. It is further observed that some joinings of the carbon nanotubes have the same quantum configuration structure irrespective of the defect following the three degeneracy states and thus obtain the same transmission for the all defective carbon nanotubes as in the figures. On the other hand, in some case one of degeneracy states gets separates out from the other degeneracy states and thus follows two different transmissions for a given pair of defective carbon nanotubes indices. One transmission obtained from one state is different for other, the same two given degeneracy states of defective carbon nanotube degeneracy states.

It is interesting to note that when two chiral metallic nanotubes are joined by different defects, the transmission is reduced in each defective carbon nanotube and even in the circumferential defective carbon nanotube case, it becomes zero. This is because the pentagonheptagons are more effective for this particular quantum configuration structure. In all type of quantum configuration structures, each defective carbon nanotubes has an effect on the transmission, but this effect is seen the least in case of circumferential carbon nanotubes and maximum in grouped defective carbon nanotubes.

\section{References}

[1] C. Dekker, Phys. Today 52, 22 (1999).

[2] S.J. Tans, A.R.M. Verschueren, C. Dekker, Nature (London) 393, 49 (1998).
[3] R. Martel, T. Schmidt, H.R. Shea, T. Hertel, Ph. Avouris, Appl. Phys. Lett. 73, 2447 (1998).

[4] P.G. Collins, A. Zettl, H. Bando, A. Thess, R.E. Smalley, Science 278, 100 (1997).

[5] Z. Yao, H.W.C. Postma, L. Balents, C. Dekker, Nature (London) 402, 273 (1999).

[6] M. Fuhrer, J. Nygard, L. Shih, M. Foreo, Y.-G. Yoon, M.S.C. Mazzoni, H.J. Choi, J. Ihm, S.G. Louie, A. Zettl, P.L. McEuen, Science 288, 494 (2000).

[7] J.W. Mintmire, B.I. Dunlap, C.T. White, Phys. Rev. Lett. 68, 631 (1992).

[8] N. Hamada, S.I. Sawada, A. Oshiyama, Phys. Rev. Lett. 68, 1579 (1992).

[9] R. Saito, M. Fujita, G. Dresselhaus, M.S. Dresselhaus, Appl. Phys. Lett. 60, 2204 (1992).

[10] T.W. Odom, J.L. Huang, P. Kim, C.M. Lieber, $N a-$ ture (London) 391, 62 (1998).

[11] D. Orlikowski, M.B. Nardelli, J. Bernholc, C. Roland, Phys. Rev. Lett. 83, 4132 (1999).

[12] M.S. Ferreira, T. Dargam, R.B. Muniz, A. Latge, Phys. Rev. B 62, 040 (2000).

[13] L. Chico, V.H. Crespi, L.X. Benedict, S.G. Louie, M.L. Cohen, Phys. Rev. Lett. 76, 971 (1996).

[14] L. Chico, L.X. Benedict, S.G. Louie, M.L. Cohen, Phys. Rev. B 54, 2600 (1996).

[15] J.C. Charlier, T.W. Ebbesen, Ph. Lambin, Phys. Rev. B 53, 11108 (1996).

[16] M. Ouyang, J.-L. Huang, C.L. Cheung, C.M. Lieber, Science 291, 97 (2001).

[17] M.S. Dresselhaus, G. Dresselhaus, P.C. Eklund, Science of Fullerenes and Carbon nanotubes, Academic Press, San Diego 1996.

[18] T.W. Odom, J.L. Huang, P. Kim, M. Ouyang, C.M. Lieber, J. Mater. Res. 13, 2380 (1998).

[19] S. Datta, Electronic Transport in Mesoscopic Systems, Cambridge University Press, Cambridge 1995.

[20] F. Guinea, J. Sanchez-Dehesa, F. Flores, J. Phys. C, Solid State Phys. 16, 6499 (1983).

[21] M.P. Lopez Sancho, J.M. Lopez Sancho, J.M.L. Sancho, J. Rubio, J. Phys. F, Met. Phys. 14, 1205 (1985).

[22] R. Nizam, S.M.A. Rizvi, A. Azam, Int. J. Selected Topics 1, 163 (2011). 